\title{
\begin{tabular}{l|l} 
Mibraries & DSpace@MIT
\end{tabular}
}

\author{
MIT Open Access Articles
}

Observation of Ion Electrosorption in Metal-Organic Framework Micropores with In Operando Small\#Angle Neutron Scattering

The MIT Faculty has made this article openly available. Please share how this access benefits you. Your story matters.

Citation: He, Lilin et al. "Observation of Ion Electrosorption in Metal-Organic Framework Micropores with In Operando Small\#Angle Neutron Scattering." Angewandte Chemie International Edition 59, 24 (April 2020): 9773-9779 (c) 2020 Wiley-VCH Verlag GmbH \& Co. KGaA, Weinheim

As Published: http://dx.doi.org/10.1002/anie.201916201

Publisher: Wiley

Persistent URL: https://hdl.handle.net/1721.1/128210

Version: Author's final manuscript: final author's manuscript post peer review, without publisher's formatting or copy editing

Terms of use: Creative Commons Attribution-Noncommercial-Share Alike 


\section{Observation of Ion Electrosorption in Metal-Organic Framework Micropores with in operando Small-Angle Neutron Scattering}

Lilin $\mathrm{He}^{*}$, Luming Yang, Mircea Dincă, Rui Zhang, Jianlin Li

Dr. L. He

Neutron Scattering Division, Oak Ridge National Laboratory, Oak Ridge, Tennessee 37831, United States

Email: hel3@ornl.gov

L.Yang, Prof. M. Dincă

Department of Chemistry, Massachusetts Institute of Technology, 77 Massachusetts Avenue, Cambridge, Massachusetts 02139, United States

Dr. R. Zhang

Energy and Mineral Engineering, Penn State University, University Park, Pennsylvania 16802, United States

Dr. J. Li

Energy \& Transportation Science Division, Oak Ridge National Laboratory, Oak Ridge, Tennessee 37831, United States

This Notice will be removed for publication:

This report has been authored by UT-Battelle, LLC under Contract No. DE-AC05-00OR22725 with the U.S. Department of Energy. The United States Government retains and the publisher, by accepting the article for publication, acknowledges that the United States Government retains a non-exclusive, paid-up, irrevocable, world-wide license to publish or reproduce the published form of this manuscript, or allow others to do so, for United States Government purposes. The Department of Energy will provide public access to these results of federally sponsored research in accordance with the DOE Public Access Plan (http://energy.gov/downloads/doepublic-access-plan). 
Abstract: Molecular-level understanding of transport and adsorption mechanisms of electrolyte ions in nanoporous electrodes under applied potentials is essential to control the performance of double layer capacitors for rapidly emerging high power energy storage. Here in operando small angle neutron scattering (SANS) is used to directly detect ion movements into the nanopores of a conductive metal-organic framework (MOF) electrode under operating conditions. Neutron scattering data reveals that most of the void space within the MOF is accessible to the solvent. Upon the addition of the electrolyte NaOTf, the ions are adsorbed on the outer surface of the protrusions to form a $30 \AA$ layer instead of entering the ionophobic pores in the absence of an applied charging potential. The changes in scattering intensity when potentials are applied suggests the ion rearrangement in the micropores following different mechanisms depending on the electrode polarization. These observations shed new insights on ion electrosorption in electrode materials. 
Graphical Abstract

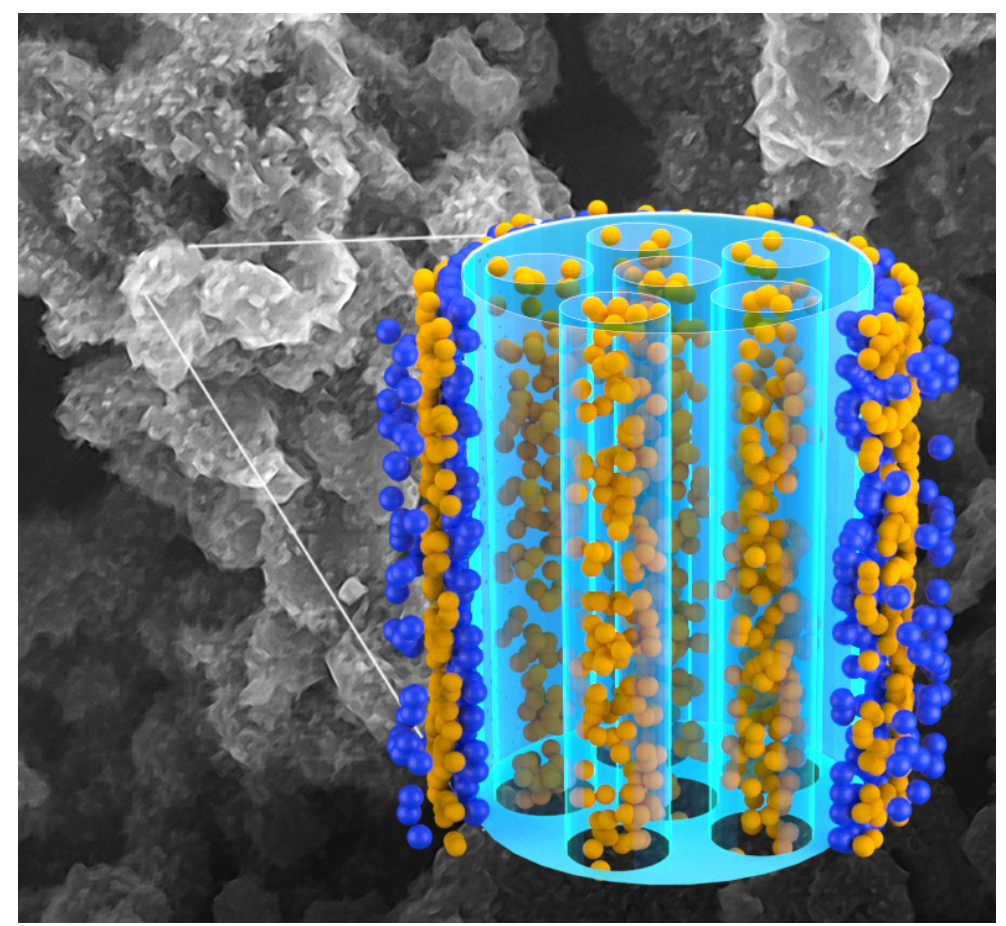

Ion arrangements in a conductive MOF electrode are examined with in operando neutron scattering. The non-destructive method provides direct and accurate information on the multilength scale morphology of the dry MOF, the pore accessibility upon soaking with solvent and electrolyte, and the changes in species confined within the micropores under operating conditions. 


\section{Introduction}

Supercapacitors (SCs) or electrical double-layer capacitors (EDLCs) represent a key element in the energy storage landscape, especially towards vehicle electrification. ${ }^{[1]}$ The fast electrosorption of ions inside the pores of the porous electrodes under an applied external voltage endows SCs significantly higher power density and longer lifetime than batteries where slow faradaic reactions or limited ion-intercalation take place. ${ }^{[2]}$ The applications of SCs have been broadened from past mundane applications into numerous industrial and consumer applications. ${ }^{[3]}$ Despite their advantages of high specific power, high energy efficiency, practically unlimited cycle life, SCs suffer from much lower energy density, particularly in comparison with Li-ion batteries. Improving SCs has led to a two-pronged approach: the development of new electrode materials and electrolytes, and designing/optimizing the morphology and porosity of existing electrode materials. Both depend critically on a deep understanding of the energy storage mechanisms, ${ }^{[4]}$ including an understanding of the relationship between the pore size and accessible surface area. ${ }^{[2,5-7]}$ It is traditionally believed that the pores should be substantially larger than the size of solvated electrolyte ions to allow the ions to diffuse into the nanopores to achieve high capacitance. However, experimental studies found that subnanometer pores could maximize the capacitance of carbide-derived carbon (CDC) materials and subsequent theoretical calculations and molecular simulations verified the anomalous phenomenon. ${ }^{[8-10]}$ The anomaly was originally ascribed to the distorted solvation or partial desolvation of counterions that lead to a closer distance between the ion center and electrode surface. ${ }^{[8]}$ It was later observed that the maximized capacitance could be achieved when the ion size matched the pore size using solvent-free ionic liquids (ILs). ${ }^{[11]}$ Huang et al. proposed a wire-in-cylinder model to take into account the effects of pore curvature on the capacitance of nanoporous carbons, which explained the observed anomalous increase in capacitance for pores below $1 \mathrm{~nm} .{ }^{[10]}$ Using molecular dynamics simulations (MD) and classical density functional theory (DFT), Feng and Jiang separately revealed that the capacitance exhibited an oscillatory mode as the pore width varied. The capacitance versus pore size was found to exhibit a strong peak for subnanometer pores. ${ }^{[12]}$ Experimental data are required to verify and validate these computer simulations.

The last decade has also witnessed the rapid development of a few in situ characterization techniques to advance the understanding of charge storage mechanism in the carbon-based supercapacitors. Among them are nuclear magnetic resonance (NMR), infrared spectroscopy 
(IR), electrochemical quartz crystal microbalance (EQCM), and small angle X-ray and neutron scattering (SAXS, SANS respectively). ${ }^{[13-16]}$ SAXS and SANS are capable of noninvasively and nondestructively studying the pore-size dependence of pore wettability and ion electrosorption based on the changes of contrast between the pore space and the pore wall while ions are injected and ejected from the pores under applied potentials. These scattering techniques combined with computer simulations have been used to quantify changes in the populations of adsorbed species during charging and provided novel insights into ion transport and charge storage mechanisms within carbon nanopores. ${ }^{[16]}$

Although considerable progress has been made by the development of advanced experimental methods and computer simulations, there are still on-going debates on the charge storage mechanisms in carbon-based electrodes due to the complexity of charging process in supercapacitors. ${ }^{[5,17]}$ According to the charging mechanism parameter introduced by Forse and co-workers, different charging mechanisms have been observed by experiments and computer simulations, which suggests that many factors, i.e. electrode polarization, voltage, electrolyte ion size and symmetry, electrode materials, pore architecture may have a synergistic impact on the charging processes. ${ }^{[5]}$ In previous reports, mesoporous carbonaceous nanomaterials have been exclusively selected as model electrode materials owning to their good electrical conductivity, tunable pore architecture and functionable surface. ${ }^{[17]}$ However, important but often ignored fact in all of these studies is that carbon electrodes were assumed to have identical slit shape in order to obtain pore-size distribution using gas adsorption and DFT methods, which is definitely problematic. ${ }^{[7,17]}$ Real carbon materials exhibit very complex features, i.e. various shapes, size distribution and different accessibility with each size range. The lack of long-range ordermakes the characterization of charge storage at the molecular level rather challenging. Therefore, nanoporous materials with uniform shape, narrow distribution pores are desirable for mechanism studies. ${ }^{[12]}$

Metal-organic frameworks (MOFs), a class of porous crystalline materials, consist of metal ions or clusters coordinated to organic ligands. They have generated a tremendous amount of interest in widespread areas ranging from electrochemical energy storage, gas storage and separation, catalyst immobilization to water desalination owing to their ultra-high porosity, well-defined and tailorable pore architectures, and functionalizable surface. ${ }^{[18]}$ The high porosity of most MOF materials, however, is inherently coupled with very poor electrical conductivity, which limits their applications as electrodes in EDLCs. Recently Sheberla et al. developed a 
conductive porous $\mathrm{MOF}, \mathrm{Ni}_{3}(\mathrm{HITP})_{2}(\mathrm{HITP}=2,3,6,7,10,11$-hexaiminotriphenylene) with an excellent electronic conductivity. ${ }^{[19,20]}$ The material is composed of stacked $\pi$-conjugated two dimensional layers, penetrated by one-dimensional cylindrical channels of $\sim 15 \AA$ diameter. It showed a loss of only $10 \%$ in capacitance and no increase in the ESR after 10,000 cycles. The surface of the MOF was $630 \mathrm{~m}^{2} \mathrm{~g}^{-1}$, as determined through $\mathrm{N}_{2}$ adsorption isotherm based on the Brunauer-Emmett-Teller (BET) theory. More interestingly, the material could serve as freestanding electrodes without additives or other binder after pressed into self-supported pellets at high pressure, which makes it an ideal material to study the ion electrosorption in micropores during the charging process. ${ }^{[19]}$

Herein, we report insights into ion arrangements within the conductive $\mathrm{Ni}_{3}(\mathrm{HITP})_{2} \mathrm{MOF}$ electrode using in operando SANS. The method provides not only direct and accurate information on the multi-length scale morphology of dry MOF and pore accessibility upon soaked with solvent, but also the changes of species confined in the micropores with and without applied potential. This study reveals that the charge storage strongly depends on the polarization of electrode. The findings observed in this work would greatly advance our understanding of the charge storage mechanisms under nanoconfinement.

\section{Results and Discussion}

All the SANS measurements in this work were carried out on the General-Purpose SANS instrument (GP-SANS) at the High Flux Research reactor (HFIR), Oak Ridge National Laboratory. The detailed experimental description can be found in the Supporting Information.

\section{Morphology of dry MOF}

A radially symmetrical 2D SANS pattern is observed for the pristine MOF sample. The raw data is azimuthally averaged to get $1 \mathrm{D}$ curve, which is shown in a log-log graph in Figure 1 (a). The curve exhibits three main regimes, arising from different levels of hierarchy of the MOF structure. In the low $q$ region of $0.007-0.06 \AA^{-1}$, the intensity decays following power law $q^{-D}$ where exponent $D$ is between 2 and 3, suggesting the mass fractal that arises from the open mass network structure resulted from the aggregation of the pores as the monomer. ${ }^{[21,22]}$ This structural feature is different than the surface fractal features frequently observed in most porous carbons. ${ }^{[23,24]} \mathrm{A}$ broad shoulder at $\sim 0.06 \AA^{-1}$ makes it transit from mass fractal to Porod scattering regime. A nearly $q^{-4}$ decay of scattering intensity in the $q$ range of $0.06-0.15 \AA^{-1}$ 
indicates relatively sharp and smooth solid matrix-pore interfaces, which allows the modelindependent evaluation of the specific surface area (SSA) based on the Porod law. ${ }^{[25]}$ The calculated SSA from the scattering curve is $\sim 871.7 \mathrm{~m}^{2} / \mathrm{g}$ considering a skeletal density of $1.1 \pm 0.2 \mathrm{~g} / \mathrm{cm}^{3}$. This value is higher than the reported Brunauer-Emmett-Teller (BET) value of $630 \mathrm{~m}^{2} / \mathrm{g}$. ${ }^{[19]}$ The discrepancy arises from two facts. One is that neutron scattering detects all the pores including open pores and close pores that probing gas molecules have no access to. The other fact is that both methods have different cutoffs of pore size to probe. ${ }^{[26]}$ The crossover from a mass fractal to a surface scattering occurs at the scattering moment corresponding to the reciprocal of the size of populated objects. In the high $q$ regime, a sharp peak located at $\sim 0.35$ $\AA^{-1}$ represents the crystalline ordering of cylindrical pores, which gives the center-to-center distance and agrees reasonably well with the pore size measured from $\mathrm{N}_{2}$ adsorption isotherm. ${ }^{[19]}$ The fitting of full curve using a summed model of fractal cylinder and a Lorentztian function indicates that the object giving rise to the scattering shoulder is about $\sim 163.7 \AA$ in length and $\sim 50.0 \AA$ in diameter (see the fitting model described in Supporting Information). These dimensions reflect the average size of meso-sized leaf-like protrusions that contain ordered micropores (see SEM image in Figure 1 (b)).

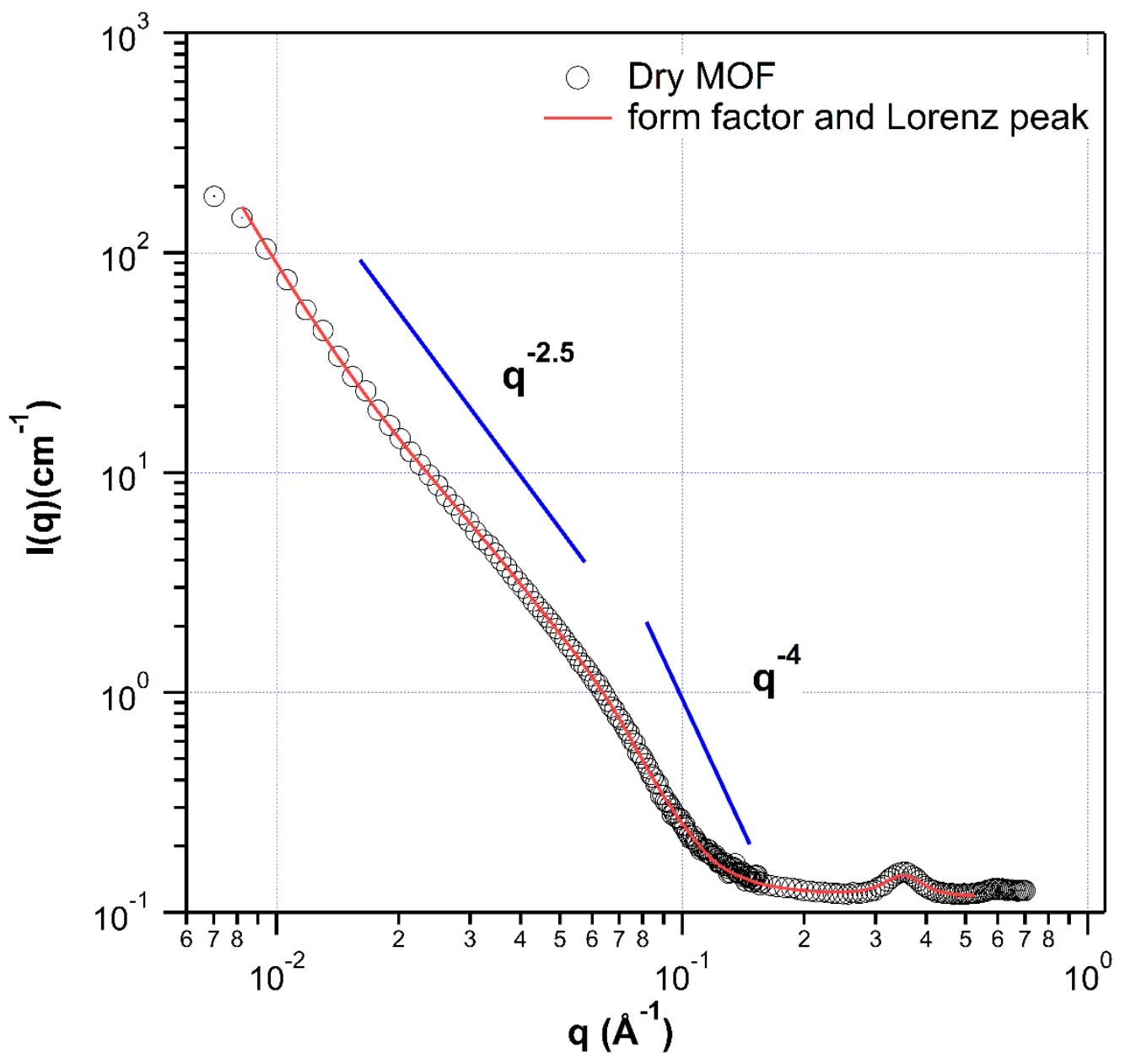


(a)

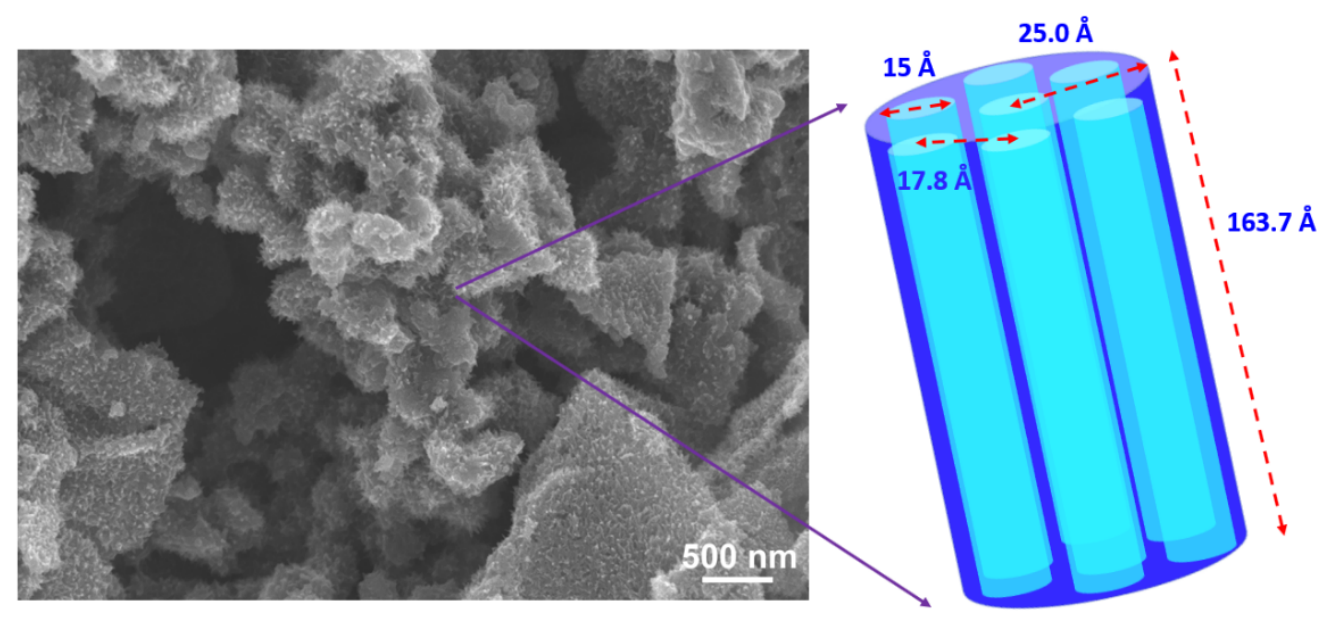

(b)

Figure 1. (a) Double logarithmic representation of the scattering intensity I(q) of the MOF electrode under dry conditions. The solid red line corresponds to the best fitting using the summed model (explained in detail in the Methods). (b) SEM image and sketch of the cylindrical protrusion. The dimensions of arranged pores were obtained based on the model fitting. The length and the radius of the leaf-like protrusions are $163.7 \AA$ and $25 \AA$, respectively. The center-to-center distance between the micropores is $17.8 \AA$. The pore size of $15 \AA$ was obtained from the gas adsorption method.

\section{Solvent penetration in $M O F$}

Upon the addition of deuterated DMF, the peak at $0.353 \AA^{-1}$ vanishes indicating that the majority of the micropores are accessible to the solvent molecules (Figure 2). Solvent wetting of carbon electrodes at the null potential has been observed in simulations. ${ }^{[7]}$ The scattering in the low $q$ region dramatically drops due to the penetration of solvent into the interstitial spaces between the protrusions and between granular particles. The scattering intensity is proportional to the contrast between the pore space and the solid matrix within the two-phase approximation:

$$
I(q) \propto\left(\rho_{M O F}-\rho_{S P A C E}\right)^{2}
$$

where $\rho_{M O F}$ and $\rho_{S P A C E}$ are neutron scattering length density (NSLD) of the MOF and the space, respectively. Assuming all these spaces are accessible to the solvent, a 98-fold drop, or $\sim 99 \%$ in percentage, in scattering intensity is expected in terms of 


$$
\frac{I_{E M P T Y}(q)}{I_{F I L L E D}(q)}=\frac{(5.753-0)^{2}}{(6.334-5.753)^{2}}=98
$$

However, the actual decrease of the scattering intensity at $\mathrm{q}=0.008 \AA^{-1}$ is $\sim 95 \%$ instead of $99 \%$, which is attributed to the pores that are inaccessible to the solvent. It is worth noting that broad shoulder near $0.06 \AA^{-1}$ also disappears due to the decreasing scattering contrast between two phases in the sample. The MOF maintains its original crystallinity structure upon exposure to the solvent, which can be seen from the SANS graph of hydrogenated DMF (Figure S2). The background level slightly increases due to the incoherent scattering of the solvent.

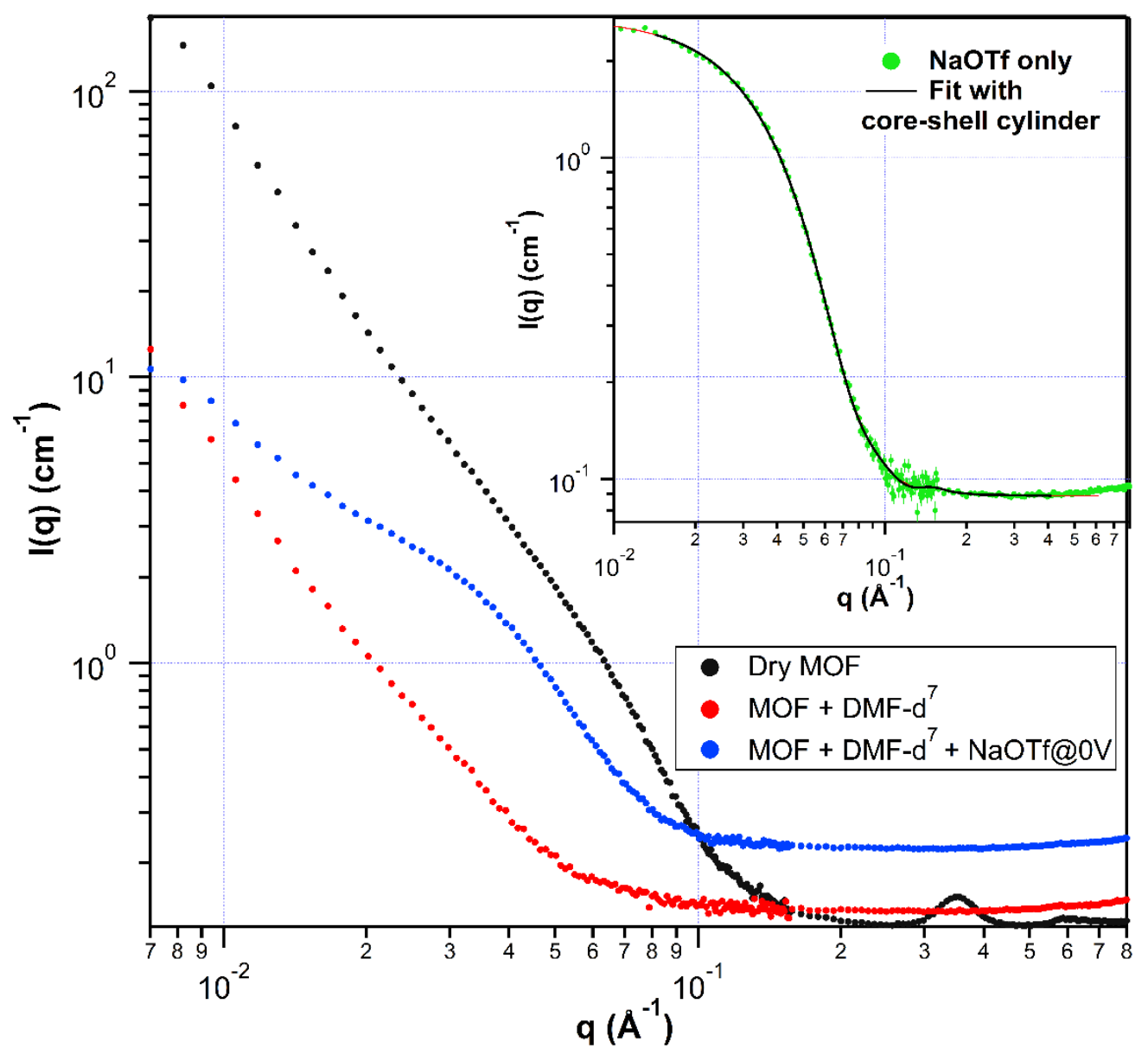

(a) 


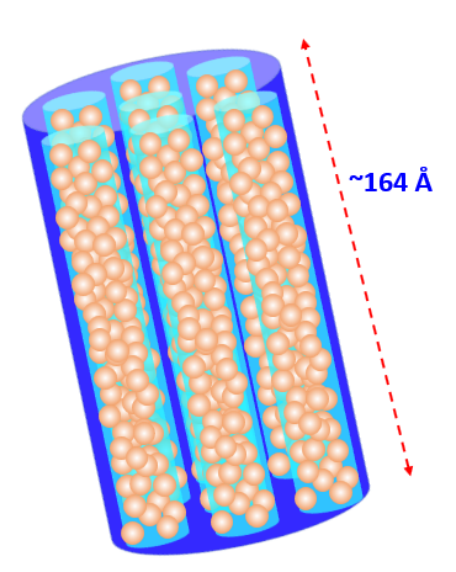

(b)

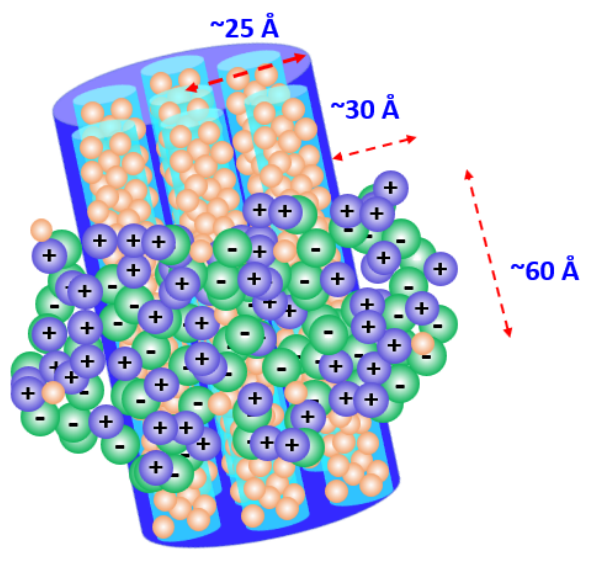

(c)

Figure 2. (a) SANS profiles of dry MOF (black), MOF with deuterated DMF (red) and MOF with electrolyte in solution (green). The inset shows the net scattering contributed by NaOTf. The solid black line in the inset corresponds to the model fitting using the core-shell cylinder form. (b) Schematic illustration the filling of solvent molecules in the MOF pores at $0 \mathrm{~V}$. (c) Ion aggregate around the protrusions to form a layer with $60 \AA$ in length and $30 \AA$ in thickness at $0 \mathrm{~V}$. The yellow balls, the purple balls, and the green balls represent solvent molecules, $\mathrm{Na}^{+}$ and OTf', respectively.

\section{Ion packing at zero applied potential}

On the SANS curve of the MOF electrode, a broad shoulder at $\sim 0.04 \AA^{-1}$ develops when the electrode is soaked with a solution of $1 \mathrm{~mol} / \mathrm{L} \mathrm{NaOTf}$ in deuterated DMF in the absence of an electric field (Figure 2 (a)). NaOTf is chosen in this work due to the significantly different NSLDs and incoherent scattering cross sections of the cation and the anion (see NSLDs in Table S1 in Supporting Information). The addition of $\mathrm{Na}^{+}$leads to an increase in incoherent scattering background (see the incoherent scattering cross sections of different species in Table S1). The net scattering signal contributed by the adsorbed cations and anions can be obtained after the subtraction of the scattering contributions of MOF and the solvent, as shown in the inset of Figure 2 (a), where the solid line corresponds to a core-shell cylinder model fitting. The fitting parameters are summarized in Table 1. The radius of $\sim 55 \AA$ for the cylinder is more than twice as large as that of the dry MOF, suggesting a large number of ions are adsorbed on the outer surface of the protrusions at the null potential to form a $\sim 30 \AA$ layer (See illustration in Figure 2(c)). However, the ionic aggregates are $\sim 60 \AA$ in length, significantly shorter than the protrusions $(\sim 164 \AA)$. This could be attributed to the fact that ion density near the end of 
each protrusion gradually decays to the bulk ion density in solution (See the illustration in Figure 2(c)). The core region shows a significantly lower NSLD than that of the shell region, which can be ascribed to the hydrogen atoms in the MOF.

\section{Ion rearrangement under applied potentials}

To investigate changes in the ion behavior under operating conditions, in operando SANS measurements were performed as the cell was sequentially charged with voltages from 0 to $+0.1 \mathrm{~V},-0.4 \mathrm{~V},+0.3 \mathrm{~V}$. The experimental setup is shown in Figure $2 \mathrm{~S}$ in the Supporting Information. The SANS profiles at these potentials are displayed in Figure 3 (a). At each potential, the data collection started full equilibration. At $+0.1 \mathrm{~V}, \mathrm{OTf}^{-}$ions enter the pores via the counter-ion adsorption mechanism, resulting in a reduction in NSLD of the core region of the core-shell cylinder, which is obtained through the model fitting using a core-shell cylinder form factor (Table 1). Both the length of the cylinder and the core radius significantly increase, whereas the shell thickness changes minimally at this potential. When the electrode is subsequently biased at $-0.4 \mathrm{~V}$, a decrease in the cylinder length and the core radius takes place, with retention of the overall shell dimension. Interestingly, the NSLD of the core further declines from that observed at $+0.1 \mathrm{~V}$. The expected behavior would be an increase of the core NSLD if $\mathrm{Na}^{+}$ions enter the pores following pure ion swapping mechanism because of the larger NSLD of $\mathrm{Na}^{+}$than that of $\mathrm{OTF}^{-}$. Presumably, during this ion exchange process, counter-ion adsorption also takes place simultaneously with expulsion of DMF- $\mathrm{d}^{7}$ out of the pores by $\mathrm{Na}^{+}$ ions. Moreover, the counter-ion adsorption process must be dominated to overcome the effect of increasing number of $\mathrm{Na}^{+}$in the pores for accounting for the eventual decreased core NSLD (Table 1). The switch of charging voltage from $-0.4 \mathrm{~V}$ to $+0.3 \mathrm{~V}$ results in increased length of the ionic cylinder and core radius as anticipated. The noticeable increase in core NSLD under this potential indicates that solvent molecules move into the pores to replace $\mathrm{Na}^{+}$ions through co-ion desorption mechanism. If the ion exchange mechanism takes places simultaneously with the co-ion desorption mechanism, the co-ion desorption has to be dominant to account for the subtle increase of the core NSLD. The majority of OTf- ions are adsorbed on the outer surface of the protrusions to balance the charge of the electrode, which is evidenced by the decreased NSLD of the shell (Table 1). The incoherent scattering background is another indicator for ions' movements under the charging process. $\mathrm{Na}^{+}$possesses a higher incoherent scattering cross section than OTf- Thus, negative potential results in an appreciably higher incoherent scattering background. 
SANS also allows exploring the species inside the micropores by observing changes of neutron scattering intensity of the $0.353 \AA^{-1}$ peak during charging. Indeed, owing to the large contrast between $\mathrm{OTF}^{-}$and the MOF wall, we would expect the correlation peak at $0.353 \AA^{-1}$ to reappear upon applying a $+0.1 \mathrm{~V}$ bias if OTf $^{-}$ions enter the pores through a pure counter-ion adsorption mechanism. However, this peak was not observed, which suggests that considerable pore space is still occupied by the DMF molecules at this potential. Presumably, large OTF- ions must overcome both an entropic barrier to enter the relatively narrow pores, and an electrostatic barrier from neighboring ions, whch is energetically unfavorable. The recurrence of the correlation peak was not observed due to the subtle change of the NSLD in the micropores.

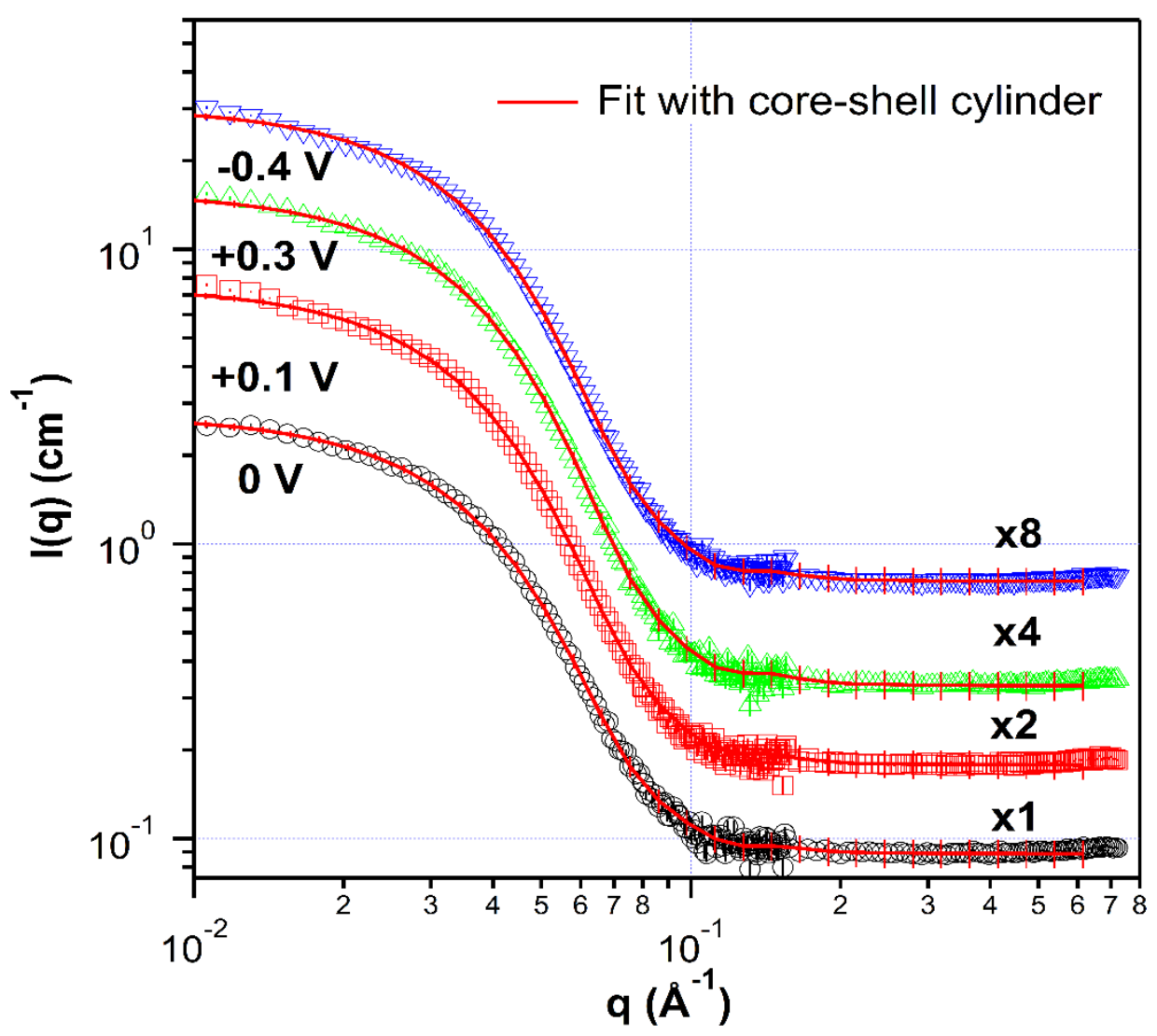

(a) 


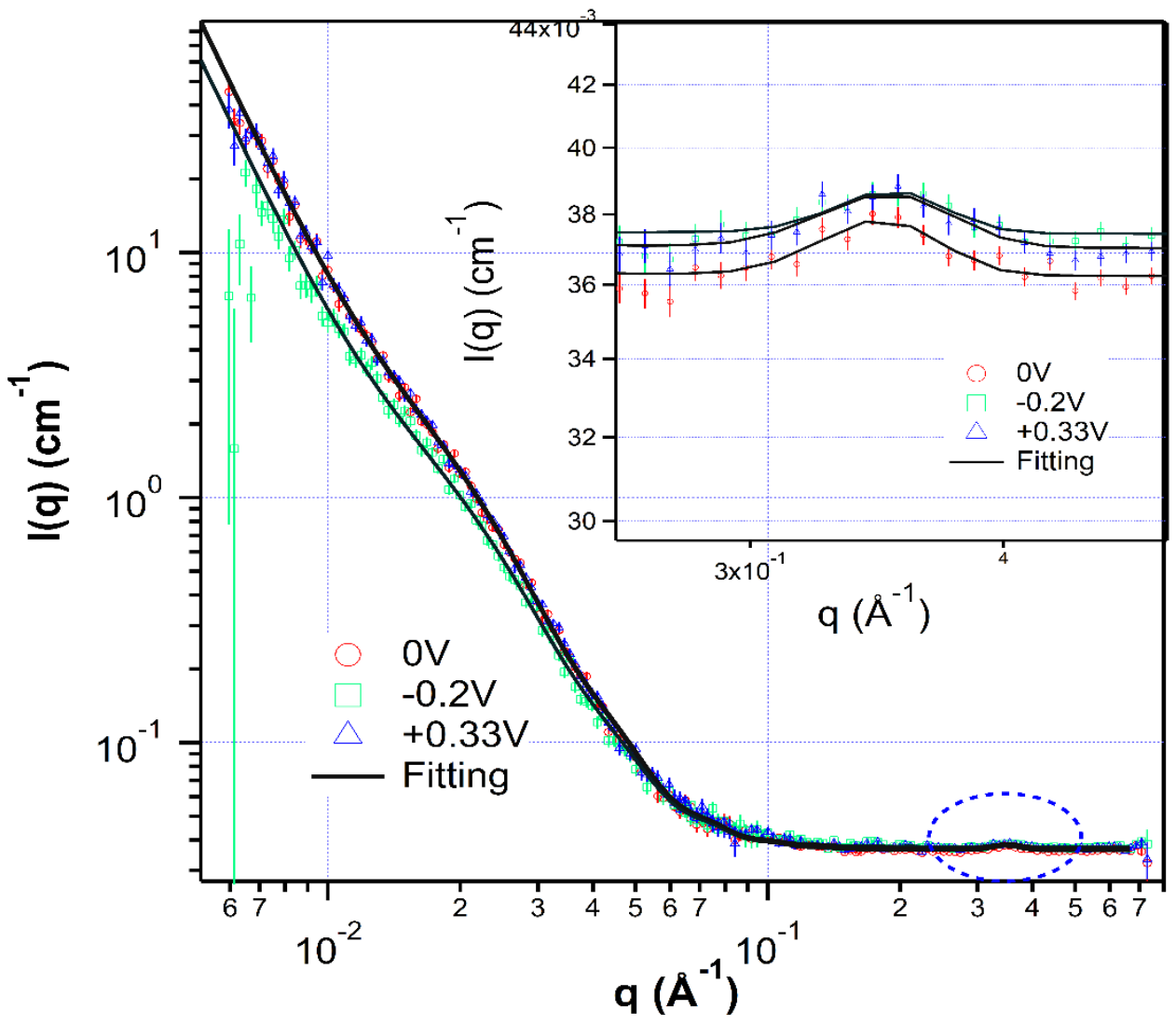

(b)

Figure 3. (a) Double logarithmic representation of MOF with electrolyte in solution at various potentials. The data are vertically shifted for visibility. The solid line in each curve corresponds to the model fitting using the cylinder form. (b) SANS profiles of the MOF electrode under three voltages. The solid line represents the fitting using a summed model with a mass fractal cylinder and a Lorentzian function. The inset plot highlights the change of peak at $0.353 \AA^{-1}$.

Table 1. Fitting parameters of SANS profiles under various potentials using a core-shell cylinder form

\begin{tabular}{ccccccc}
\hline $\begin{array}{c}\text { Potential } \\
(\mathrm{V})\end{array}$ & $\begin{array}{c}\text { Core Radius } \\
(\AA)\end{array}$ & $\begin{array}{c}\text { Shell } \\
\text { Radius }\end{array}$ & $\begin{array}{c}\text { Cylinder } \\
\text { Length }\end{array}$ & $\begin{array}{c}\text { Core } \\
\text { NSLD } \\
(\AA)\end{array}$ & $\begin{array}{c}\text { Shell } \\
\text { NSLD }\end{array}$ & $\begin{array}{c}\text { I }_{\text {inc }} \\
\left(\mathrm{cm}^{-1}\right)\end{array}$ \\
\hline 0 & & $\left(\times 10^{-6} \AA^{-2}\right)$ & $\left(\times 10^{-6} \AA^{-2}\right)$ & \\
+0.1 & $26.93 \pm 0.45$ & $29.58 \pm 0.22$ & $60.44 \pm 1.68$ & $4.69 \pm 0.02$ & $6.07 \pm 0.01$ & $0.0889 \pm 0.0001$ \\
-0.4 & $25.06 \pm 0.29$ & $30.45 \pm 0.14$ & $61.68 \pm 1.07$ & $4.44 \pm 0.02$ & $6.03 \pm 0.01$ & $0.0931 \pm 0.0001$ \\
+0.3 & $25.13 \pm 0.40$ & $29.78 \pm 0.21$ & $62.87 \pm 1.53$ & $4.47 \pm 0.02$ & $6.02 \pm 0.01$ & $0.0824 \pm 0.0002$ \\
\hline
\end{tabular}


To further investigate the changes of species inside the micropores, we subsequently employed a coin cell setup (Figure 3S in Supporting Information) to monitor ion movements in the micropores during the charging process. The schematic picture shows the assembly of the cell. In these measurements, we used hydrogenated DMF instead of deuterated DMF by taking advantage of the isotope sensitivity of neutrons. The thin sample inside of coin-cell $(\sim 1 \mathrm{~mm})$ allows for the use of h-DMF by minimizing multiple scattering and incoherent scattering from the hydrogens in the solvent. The height and width of the peak located at $0.353 \AA^{-1}$ are sensitive to the organization of electrolyte and solvent molecules inside the micropores. Figure 3(b) displays the SANS profiles with changing the potential. A Lorentzian model was used to fit the peak and the fitting parameters are listed in Table 2.

Table 2. Changes in micropores under potentials

\begin{tabular}{llll}
\hline $\begin{array}{l}\text { Potential } \\
(\mathrm{V})\end{array}$ & $\begin{array}{l}\text { Peak Height } \\
\left(\times 10^{-3}\right)\end{array}$ & $\begin{array}{l}\text { Peak Position } \\
\left(\AA^{-1}\right)\end{array}$ & FWHM \\
\hline 0 & $1.59 \pm 0.19$ & $0.348 \pm 0.003$ & $0.023 \pm 0.003$ \\
-0.20 & $1.26 \pm 0.22$ & $0.352 \pm 0.004$ & $0.022 \pm 0.004$ \\
+0.33 & $1.57 \pm 0.21$ & $0.351 \pm 0.004$ & $0.026 \pm 0.004$ \\
\hline
\end{tabular}

When charging the electrode under $-0.20 \mathrm{~V}$, a drop in peak height from 1.59 to 1.26 indicate that $\mathrm{Na}^{+}$ions enter the micropores via counter-ion adsorption mechanism, which decreases the contrast between the pore and the solid wall. Under the positive potential of $+0.33 \mathrm{~V}$, on the contrary, $\mathrm{Na}^{+}$ions in the micropores leave the pores following combined mechanisms of ion exchange and co-ion desorption, which results in a larger contrast between the pore and the wall. Therefore, an increase in the peak height is observed in comparison with that at the negative polarization (Table 2). This is consistent with our previous observations. The different charging mechanisms at different polarization conditions are illustrated in Figure 4. 


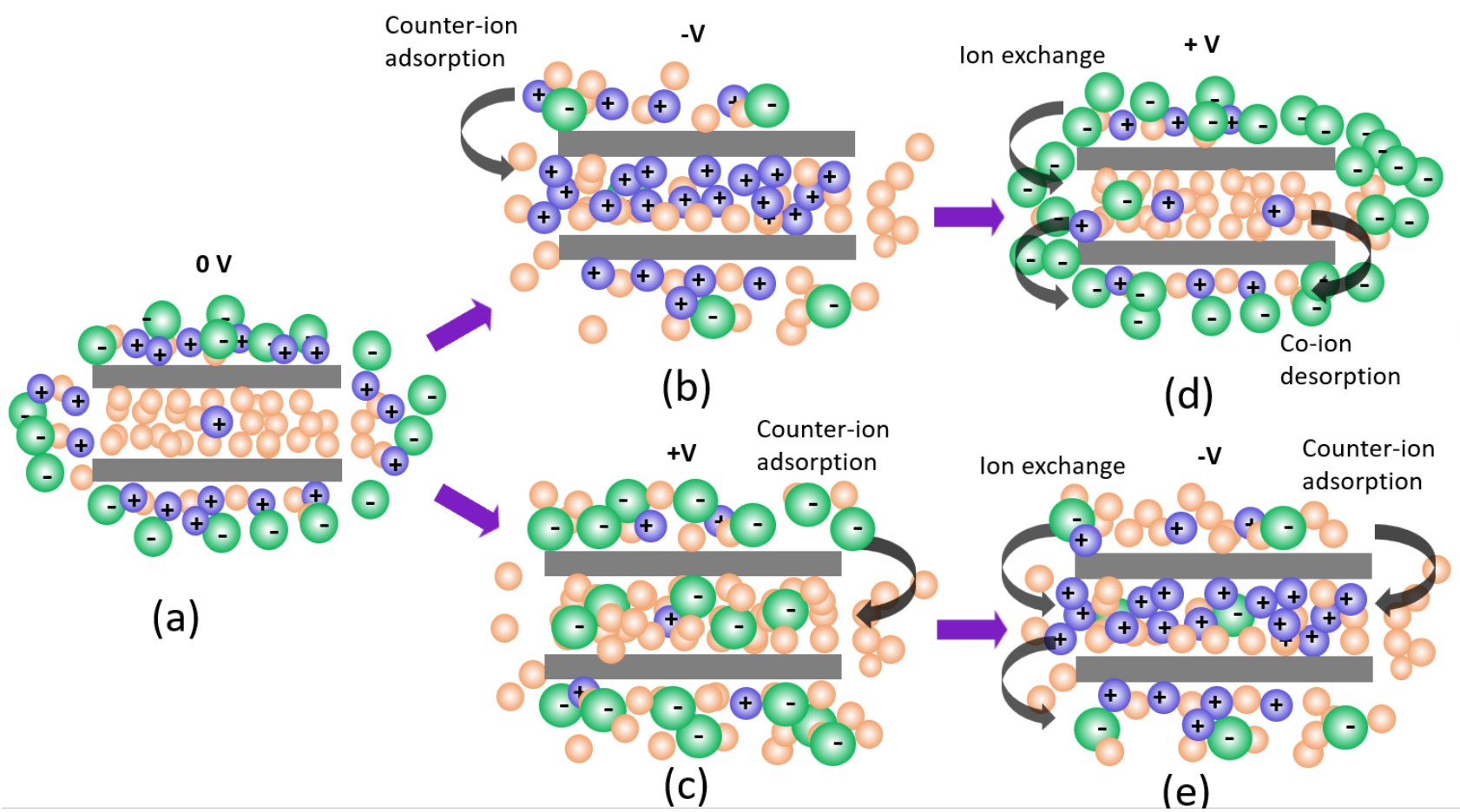

Figure 4. Scheme of different charging mechanisms for ionophobic MOF micropores depending on the polarization of the electrode. The yellow balls, the purple balls, and the green balls represent solvent molecules, $\mathrm{Na}^{+}$and $\mathrm{OTF}^{-}$, respectively. (a) At $0 \mathrm{~V}$, the ionophobic pores are filled with solvent molecules. (b) and (c) From $0 \mathrm{~V}$ to a negative potential or positive potential, the charging goes majorly through counter-ion adsorption. (d) Switch the potential from negative to positive, co-ion $\left(\mathrm{Na}^{+}\right)$desorption dominates, ion exchange mechanism may take place simultaneously. (e) Switch the potential from positive to negative, counter-ion $\left(\mathrm{Na}^{+}\right)$ adsorption dominates, ion exchange mechanism could occur simultaneously.

If the micropores are ionophilic and $\mathrm{Na}^{+}$ions are present in the pores at the null potential, we should anticipate a greater peak height at $+0.33 \mathrm{~V}$ compared with that at $0 \mathrm{~V}$ once most of these initial $\mathrm{Na}^{+}$ions leave the pores. However, the peak heights at $0 \mathrm{~V}$ and at $+0.33 \mathrm{~V}$ are comparable (Table 2), which suggests that the micropores have only negligible amount of $\mathrm{Na}^{+}$ions at $0 \mathrm{~V}$. Kondrat et al. have described two kinds of pores in porous electrodes: "ionophilic" and "ionophobic" in their simulation. Their results showed that electrodes with ionophobic pores could facilitate fast charging and suggested that the energy capacity is higher for materials with ionophobic pores. ${ }^{[27,28]}$ This is the first time to experimentally observe ionophobic pores, to our best knowledge. The center-to-center distance between the micropores, reflected by the peak position, indicates no noticeable expansion of the pores. The peak broadening, coming from both lattice imperfection and instrument resolution and indicated by full width at half 
maximum (FWHM), shows negligible change revealing that the material retains its ordered arrangement during charging process.

\section{Conclusions}

We have used in operando SANS to probe ion electrosorption into a conductive MOF. The dry MOF is composed of cylindrical protrusions with embedded micropores. Most of the space within the MOF including micropores and interstitial volume are accessible to the solvent. Electrolyte ions are adsorbed on the outer surface of the protrusions instead of entering the micropores at null potential. Our results also indicate that the ionophobicity of the micropores causes pure counter-ion adsorption when applying a nonzero bias voltage. Subsequent charging process exhibits different storage mechanisms strongly depending on the polarization of electrode. When switching from negative to positive polarization the process proceeds predominantly through the co-ion desorption mechanism, whereas switching from a positive to a negative polarization, the charging process majorly follows the counter-ion adsorption mechanism. These results provide molecular level insight into the charging mechanisms in the MOF electrode.

\section{Supporting Information}

Supporting Information is available free of charge on the ACS Publications website at DOI: Material characterization using static SANS, powder X-ray diffraction, electrode preparation, in operando SANS experiment, SANS data analysis and neutron scattering length densities and incoherent scattering cross sections of chemical species.

\section{Author contributions}

L.H. pursued the idea. L. Yang synthesized the MOF, L. H., L. Yang, R. Zhang, J. L. performed SANS experiment, L. H. wrote the manuscript while L. Yang, R. Zhang, J. Li, and M. Dinca were involved in the technical discussions and manuscript preparation.

\section{Conflict of interest}

The authors declare no competing financial interest. 


\section{Keywords}

metal-organic frameworks, supercapacitor, small angle neutron scattering, microporous materials, electrosorption

\section{Acknowledgements}

This research used resources at the High Flux Isotope Reactor, DOE Office of Science User Facilities operated by the Oak Ridge National Laboratory. Research sponsored by the Laboratory Directed Research and Development Program of Oak Ridge National Laboratory, managed by UT-Battelle, LLC, for the U. S. Department of Energy.

\section{References}

[1] F. Beguin, F, V. Presser, A. Balducci, E. Frackowiak, Adv. Mater. 2014, 26, 2219-2251.

[2] M. Salanne, B. Rotenberg, K. Naoi, K. Kaneko, P. L. Taberna, C. P. Grey, B. Dunn, P. Simon, Nat. Energy 2016, 1, 16070.

[3] Y. G. Wang, Y. F. Song, Y. Y. Xia, Chem. Soc. Rev. 2016, 45, 5925-5950.

[4] G. P. Wang, L. Zhang, J. J. Zhang,Chem. Soc. Rev. 2012, 41, 797-828.

[5] A. C. Forse, C. Merlet, J. M. Griffin,C. P. Grey, J. Am. Chem. Soc. 2016, 138, 5731-5744.

[6] F. X. Wang, X. W. Wu, X. H. Yuan, Z. C. Liu, Y. Zhang, L. J. Fu, Y. S. Zhu, Q. M. Zhou, Y. P. Wu, and W. Huang, Chem. Soc. Rev. 2017, 46, 6816-6854; A. Karatrantos and Q. Cai, Phys. Chem. Chem. Phys. 2016, 18, 30761-30769.

[7] C. Zhan, C. Lian, Y. Zhang, M. W.Thompson, Y. Xie, J. Z. Wu, P. R. C. Kent, P. T. Cummings, D. E. Jiang, D. J. Wesolowski, Adv. Sci. 2017, 4, 1700059.

[8] J. Chmiola, G. Yushin, Y. Gogotsi, C. Portet, P. Simon, P. L. Taberna, Science 2006, 313, 17601763.

[9] P. Simon and Y. Gogotsi, Phil. Trans. R. Soc. A 2010, 368. 3457-3467; X. H. Wang, A. Y. Mehandzhiyski, B. Arstad, K. L. Van Aken, T. S. Mathis, A. Gallegos, Z. Q. Tian, D. D. Ren, E. Sheridan, B. A. Grimes, D. E. Jiang, J. Z. Wu, Y. Gogotsi, and D. Chen, J. Am. Chem. Soc. 2017, 139, 18681-18687.

[10] J. S. Huang, B. G. Sumpter, V. Meunier, Angew. Chem. Int. Edit. 2008, 47, 520-524.

[11] C. Largeot, C. Portet, J. Chmiola, P. L. Taberna, Y. Gogotsi, and P. Simon, J. Am. Chem. Soc. 2008, $1302730-2731$.

[12] G. Feng,P. T.Cummings, J. Phys. Chem. Lett. 2011, 2, 2859-2864; D. E. Jiang, Z. H. Jin, J. Z. Wu, Nano. Lett. 2011, 11, 5373-5377.

[13] M. Deschamps,E. Gilbert, P. Azais, E. Raymundo-Pinero, M. R. Ammar, P. Simon, D. Massiot, F. Beguin, Nat. Mater. 2013, 12, 351-358; H. Wang, T. K. J. Koster, N. M. Trease, J. Segalini, P. L. Taberna, P. Simon, Y. Gogotsi, and C. P. Grey, J. Am. Chem. Soc. 2011, 133, 1927019273.

[14] F. W. Richey, B. Dyatkin, Y. Gogotsi, Y. A. Elabd, J. Am. Chem. Soc. 2013, 135, 1281812826.

[15] M. D. Levi, G. Salitra, N. Levy, D. Aurbach, J. Maier, Nat .Mater. 2009, 8, 872-875.

[16] S. Boukhalfa, L. L. He,Y. B. Melnichenko, G. Yushin, Angew. Chem. Int. Edit. 2013, 52, 46184622; C. Prehal, D. Weingarth, E. Perre, R. T. Lechner, H. Amenitsch, O. Paris, V. Presser,Energ. Environ. Sci. 2015, 8, 1725-1735.

[17] A. Garcia-Gomez, G. Moreno-Fernandez, B. Lobato, and T. A. Centeno, Phys. Chem. Chem. Phys. 2015, 17, 15687-15690; T. A. Centeno, O. Sereda, and F. Stoeckli, Phys. Chem. Chem. Phys. 2011, 27, 12403-12406; A. Eftekhari, Mater. Today Chem. 2018, 7, 1-4.

[18] H. B. Wu, X. W. Lou, Sci. Adv. 2017, 3, eeap9252; G. Y. Xu, P. Nie, H. Dou, B. Ding, L. Y. Li, X. G. Zhang, Mater. Today 2017, 20, 191-209; H. Furukawa, K. E. Cordova, M. O'Keeffe, O. M. Yaghi, Science 2013, 341, 1230444. 
[19] D. Sheberla, J. C. Bachman, J. S. Elias, C. J. Sun, Y. Shao-Horn, M. Dinca,Nat. Mater. 2017, $16,220-224$.

[20] D. Sheberla, L. Sun, M. A.Blood-Forsythe, C. R. Wade, Er, C. K. Brozek, A. Aspuru-Guzik, M. Dinca, J. Am..Chem. Soc. 2014, 136, 8859-8862.

[21] P. McMahon, I. Snook, J. Chem. Phys. 1996, 105, 2223-2227.

[22] D. F. R. Mildner. P. L. Hall, J. Phys. D: Appl. Phys. 1986. 19, 1535-1545.

[23] H. D. Bale, P. W. Schmidt, Phys. Rev. Lett. 1984, 53, 596-599.

[24] L. L. He, Y. B. Melnichenko, N. C. Gallego, C. I. Contescu, J. J. Guo, and J. Bahadur, Carbon 2014, $80,82-90$.

[25] O. Glatter, O.Kratky, Small angle x-ray scattering. New York: Academic Press,, London 1982.

[26] L. M. Anovitz, D. R. Cole, Rev. Mineral. Geochem. 2015, 80, 61-164.

[27] S. Kondrat, P. Wu, R. Qiao, A. A. Kornyshev, Nat. Mater. 2014, 13, 387-393.

[28] S. Kondrat, A. A. Kornyshev, Nanoscale Horiz. 2016, 1, 45-52. 